\title{
Editorial
}

\section{Issue of Mental health at our work place}

DR Shakya, Department of Psychiatry

BP Koirala Institute of Health Sciences, Dharan

A significant part of a day and entire life of an individual of this era is spent in his/ her work place, station or field. ${ }^{1}$ There are factors in particular office, workplace or field which exert protective effect to the holistic health of the related stakeholders and empower them. Similarly, there may be some risk factors leading to ill health, lost peace and failure in life. These factors not only affect body, but also mind and brain, mental direction, psychological state and mental health as a whole. The circumstances, environment and exchanges in the work place interact not only psycho-socially, but also biologically in a complex way, either to protect or risk an individual from ill health. Mental illness affects a significant proportion of any population ${ }^{2}$ but many of them remain undetected and unattended. ${ }^{3}$

The morbidity, disability and mortality due to suicide and neglect of overall health because of psychiatric illness remain high. The unattended/ untreated illness also results in reduced working capacity and skills, decline in economical status and

\footnotetext{
Address for correspondence

Dr. Dhana Ratna Shakya, MD

Professor

Department of Psychiatry

BP Koirala Institute of Health Sciences, Dharan

Email: drdhanashakya@yahoo.com
}

overall productivity. The cost incurred due to chronic illness also contributes to economic drop. Mental disorder spares none of us; staff, workers, teachers, students, farmers, managers, leaders, policy makers and all. It affects though in some varying proportions depending on the risk and protective factors within and around us, including those in our workplace. Hence, there is a need for an employee, employer, manager and organization to closely observe this interaction. Are we conscious and concerned enough about this issue? Here, we intend to raise and draw attention of related stakeholders towards this often forgotten (in our context) but important issue.

Our institute, B. P. Koirala Institute of Health Sciences (BPKIHS) was established on Jan 18, 1993 with main objective of developing socially responsible and competent health workforce, providing health care and involving in innovative health research. ${ }^{4}$ This has been work place directly to a total of 1648 staff including faculty, other teachers, administrative and other staff (1008 on permanent, 99 performance, 541 contract basis) and an educational centre for a total of 1598 students of various programs and levels in this academic year 
of 2017/18 AD (BPKIHS, December 2017). ${ }^{5}$ These stakeholders; not only the buildings, roads, lands here are the basis of the direction of, move towards, progress and achievement of these objectives. Only with this complementing appreciation and accordingly the behavior among the stakeholders (authority, staff, workers, teachers, students), we will be able to achieve the goal.

Medical and education fields, both are sensitive and stressful. Now, it is high time to look into our local contexts, circumstances and stressor status of both medical and non-medical staff of this institute. We, as a part of this institute, leading in the country should strive to set an example in the direction of mental health friendly work place. For this need of the era, let's unite and work step by step.

First thing, we need to start in this direction, is the information and data regarding overall health status including mental disorder and stressor, health indicators, local contexts and circumstances, risk and protective factors. Realistic analysis, sincere approach and appropriate management are as equally important as timely increment of salary for the rise of happiness index and quality of life here. Time has come now for all the related sides; the institute, authority, teachers, staff and students to take their respective role of resource mobilization, coordination, research conduction and participation for generation of such operational research data.

Second equally important component is mental health friendly policy and plans. Whole country is involved these days in writing and revising the Constitution of Nepal. Let's not forget that only with mental peace and health of its stakeholders, we will be able to move this institute forward.

It is not only important to bear respective role at institute level, but also at individual level. Lets we teachers, health professionals, staff, students, all service providers introspect ourselves whether we have open and healthy communication, respectful and civilized manners, empathetic and supportive behaviors among ourselves and with service users. ${ }^{1}$ Are we encouraging behaviors or cultures inviting ill health, like rampant use of alcohol, cannabis or displaying wasteful expenses in parties and celebrations? Are we secluding ourselves and our children sparing from our healthy cultures, 
festivals, occasions and traditions, and indiscriminately indulging in internet and social media in the name of modernity and advancement?

No one from outside will bother whether our working environment, residential settings and places are safe (e.g. humps with no coloring and adequate light), healthy, peaceful; free from pollution (noise, air, water, soil) or having adequate lighting and comfortable temperature etc. and free from or with minimum of occupational hazards. There is no alternative to our own sincere concern.

We have enough evidences indicating that many and many people are affected by mental agony, ailments and stress. We are not the exception. ${ }^{2,3}$ But, are we well informed, aware, alert and concerned about this? Let's consider and accept this fact and develop positive attitude. We need regular awareness raising programs for all stakeholders. Let's review whether we have a mechanism, process and unit to ensure that our needy people (with stress, problem, issue or disorder) are appropriately heard, attended and helped. Are we adopting compatible view to our colleagues in our own work place struggling or recuperating from stress and mental illness?

Continuous review is required regarding whether our departments, units and offices are compatible to the interest, skill, subjects, training, post/ designation of its staff. Transparent review on the equitable distribution and provision of opportunity for training, education and career development is paramount for both individual and academic organization. At organizational levels, let's consider whether our work place, burden and schedules are overburdened and stressful or whether less stimulating, too boring or too leisurely. At individual level, let's be watchful whether any of us are displaying warning signs of stress, ill health or mental disorder. Let's help each other and facilitate seek help from the expert. It's the high time now to think sincerely about mental health at workplace. ${ }^{6}$ May we not be left behind!

The intention of raising this issue here in this journal is to support brain storming, introspection, reflection, and motivation for clear direction, expression and execution of mental health friendly workplace philosophy. 


\section{References}

1. Gray P. Mental health in Workplace. Mental Health Foundation, Victoria Street, London, UK. 1999. P. 1.

2. Murray CL, Lopez AD. The Global Burden of Disease: a Comprehensive Assessment of Mortality and Disability from Diseases, Injuries, and Risk Factors in 1990 and Projected. Cambridge, Mass: Harvard University Press; 1996.

3. Wang PS, Aguilar-Gaxiola S, Alonso $J$, et al. Use of mental health services for anxiety, mood, and substance disorders in 17 countries in the WHO world mental health surveys. Lancet. 2007; 370: 841-50.

4. B. P. Koirala Institute of Health Sciences. Available athttp://bpkihs.edu/introduction.html, Accessed at December 24, 2017.

5. B. P. Koirala Institute of Health Sciences. Annual Report 2016-17 and Plan of Action 2017-18. BPKIHS, Dharan, Nepal. 2017.

6. World Health Organization. World Mental Health Day 2017. Available atwww.who.int/mental_health/worldmental-health-day/2017/en/. Accessed at September 11, 2017. 